\title{
Future of the pharmaceutical industry in the GCC countries
}

\author{
Abdulaziz Alsaddique* \\ VP CCIC Group AND CEO Qimat Taiba Pharmacuetical Biotechnolgy Factory, Saudi Arabia
}

\begin{abstract}
Pharmaceutical Industry in the Gulf Cooperation Council (GCC) Countries started some 25 years ago. The main idea was to decrease cost of the medications which was increasing rapidly. Some of the GCC Countries have started manufacturing pharmaceuticals products under the license of major Pharmaceutical companies. The purpose was to get quality products and developing the know-how as well as the potential of technology transfer. Soon enough they discovered the Generics saving and raw materials can be purchased much cheaper than from the major Pharmaceutical companies which they manufacture for. The GCC pharmaceutical plants practically kicked off their partners and moved into the line of Generics, competing with the major companies.

The GCC Pharmaceutical companies got some success in reducing the prices of the raw materials but this was at the expense of the quality of their products. The quality of Generics so far failed to match the Brand products. The GCC companies are still competing for the same line of products among and between the other GCC countries. The GCC Countries still import $90 \%$ of their drug needs from the major international companies. Biotechnology will never be different than the other Pharmaceutical products. There are limited efforts to establish a Pharmaceutical Biotechnology manufacturing in some of the GCC countries where the main products in mind are Insulin, Hormones, and Vaccines.
\end{abstract}

I don't believe that the efforts in the GCC with Biotech products will be any different from that on the other Pharmaceuticals. The GCC needs to join forces and set Research based facilities, try to integrate their manufacturing, and work as one team for one Goal.

\section{Introduction}

The pharmaceutical market in the Gulf Cooperation Council (GCC) has witnessed considerable progress over the years due to favorable demographic and economic factors, and strong government support for healthcare. Despite the progress, the pharmaceutical sector in the Gulf is still in an emerging phase, and drug manufacturing is at a relatively nascent stage due to a number of obstacles. A vast majority of pharmaceuticals consumed in the region are of imported brand. However, the governments have been trying to increase local drug manufacturing and reduce reliance on imported products by encouraging joint ventures and licensing deals with multinational pharmaceutical companies.

The Gulf countries are expected to spend USD 12 billion on the pharmaceutical industry by 2020 and Saudi has the largest market for medication in the region of USD 5.1 billion in 2012. Saudi Arabia's per capita share of drugs sale is USD 175 yearly despite the support of the government for the health care [1]. It is expected that investment in Healthcare in the Middle East will reach USD 60 billion by 2025, especially that these countries need between 120 and 140 additional hospitals by 2020 to meet the increased demand [2].

Comparing expenditure on health care, Saudi spends the most, followed by Egypt, United Arab Emirates (UAE), Kuwait, and Qatar, without looking at the country population. The size of the pharmaceutical industry by these countries reached USD 18 billion by 2012, compared to USD 16 billion in 2011 and USD 12 billion in 2008, with is an annual growth of $10 \%$ [2].

A recent report also showed that total spending on health care projects, including plans to manufacture drugs, in the Middle East reach USD 125 billion in 2015 [3].
Despite the fact that Pharmaceutical industry started long time ago in the GCC countries, yet it is still importing more than $90 \%$ of its Pharmaceuticals from international companies. In this paper we will try to shed some of the light on the obstacles that the GCC Pharmaceutical companies facing in its development and creating a solid ground for its development and growth.

\section{Pharmaceutical industry in the GCC countries}

The size of the pharmaceutical industry reached USD 8.5 billion by the end of 2012, compared to USD 7.7 billion in 2011. Saudi produces $59.4 \%$ of medicine in the region, followed by $18 \%$ in UAE, 9.2\% in Kuwait, $5.6 \%$ in Oman, $4.5 \%$ in Qatar and finally $3.1 \%$ in Bahrain [4]. Health care spending in the GCC will increase as the sector grows, which will lead to a decrease in the percentage spent on pharmaceuticals compared to the total health care spending to match those of the developed world, expecting a decrease from $14.3 \%$ in 2010 to $12.4 \%$ by 2020 [2].

The trend in consuming medication is changing due to changes in how diseases are spread. Urban development and the increase per capita income in the GCC lead to a more comfortable lifestyle and increased unbalanced diets. This leads to an increase in health problems

Correspondence to: Abdulaziz Alsaddique Pharm.D., CPHQ, SSMBB, VP CCIC Group AND CEO Qimat Taiba Pharmacuetical Biotechnolgy Factory, Saudi Arabia, E-mail: drsaddique1@hotmail.com

Key words: pharmaceutical, GCC, manufacturing, integration, privatization, corporatize

Received: July 02, 2017; Accepted: July 24, 2017; Published: July 27, 2017 
such as diabetes, heart and cardiovascular diseases, which will affect the pharmaceutical industry as well as health care costs [2].

Recently there are efforts to pursue international companies to invest in the GCC countries. There are between 15 to 20 local and foreign pharmaceutical manufacturers in Saudi. Last November, Modern Industrial Investment Holding Group, the Malaysian Pharmaniaga and the American E-Healthline announced signing a memorandum of agreement to build a pharmaceutical industrial complex in Saudi for SAR 600 million (USD 160 million). The total revenue from pharmaceuticals in Saudi increased by $11.3 \%$ between 2008 and 2010, according to the report that confirmed a decrease in exported pharmaceuticals due to the increase in local demand [2]. This increased focus on the development of the pharmaceutical industry has motivated and encouraged particular international companies to become more involved in the UAE pharmaceutical industry. Some are looking to start obtaining the necessary approvals from the relevant regulators to establish Research and Development (R\&D) Laboratories or manufacturing plants in the UAE [5].

Recently the UAE established a Biotech factory in collaboration with GL Rapha and Hankook Korus Pharm, two pharmaceutical companies based in Seoul, South Korea, to construct the factory and market the products [6]. On the other hand, Julphar Company in the $\mathrm{UAE}$ is also developing its biosimilar biotechnology Pharmaceutical production [7].

Pharmaceutical Biotechnology is an expensive investment, USA invested in 2005, $\$ 12.8$ billion in R\&D, while Europe's biotech sector invested only $\$ 2.7$ billion. Likewise, China's R\&D “intensity” the ratio of an organization's expenditures on R\&D to its sales grew 17 times more than the European Union's between 1999 and 2003 [8]. At that time, you could barely hear about biotechnology in the Arab World that developed an R\&D. Practically most of the companies are looking for Biosimilar. This is an alarming attitude, which will take us back to the same practice on the other Chemical Pharmaceuticals. It is believed that high investment in R\&D does not produce the expected fast cash and revenues as with Biosimilar.

Biotechnology should be considered as a national security for the country. The government should be give utmost priority and as much needed support to secure the industry. With the understanding of the high investment cost in this industry, but is getting to be essential for practically all countries in the world.

The GCC countries are considering seriously the development of Biotechnology industry. They have facilitated foreign investment and signed numerous agreements with international companies. The concern here is whether the industry will flourish as expected, or it will be initiated strongly and then gradually the concern for fast income will dominate, following the same steps of the Chemical Pharmaceuticals. Another concern arise, that is; will the biotechnology factories will be complementing one another, or they will be competing for the same products and neglect other products with lower income? In other words, will they follow the Blue Ocean Strategy or the Red Ocean one?

History gives a clear picture. 30 years since the development of Chemical Pharmaceutical industry and barely achieved $10 \%$ of the GCC needs. Manufacturers were competing for the very same products. There are more than 40 Pharmaceutical companies in the GCC countries, and we are still relying heavily on the multinational companies [9].

\section{Challenges to the industry in the gulf}

There are many challenges to manufacturing pharmaceuticals in the GCC, such as the lack of expertise and skilled manpower and the continuous changing of the business rules and regulations, which limits the growth of the industry and increases the reliance on foreign supply. Another problem is the spread of fake drugs in the GCC countries, and prices being higher than the global average. Furthermore, Gulf countries rely primarily on importing pharmaceutical manufacturing equipment, and pharmaceutical ingredients and medicines.

The previously mentioned Alpen Capital report confirmed that the GCC revenue from imported drugs is much higher than from the local production that does not exceed $10 \%$. The Gulf manufacturers are primarily focusing on producing general medication and are having difficulty in competing with foreign companies. Perhaps, the limited local production and its inability to meet the increasing demand make this sector in the Gulf more attractive for private investors.

\section{The future of pharmaceutical industry in the GCC}

The pharmaceutical industry in the gulf is still in the early development stages compared to international standards. Despite that, it is changing through reform and simplifying government regulations, increasing its efficiency and expanding the infrastructure of health care [2]. Based on that, the pharmaceutical industry in GCC will face many changes in the next few years, which will provide important investment opportunities. The local production is expected to increase. Thanks to the increase of foreign investment in this sector.

Population growth in the GCC will be a key growth driver for the pharmaceutical sector. Population is anticipated to expand from 37.5 million in 2007 to nearly 50 million in 2017. High levels of urbanization and a strong expatriate presence also support pharmaceutical sales growth in the region. Population aged 60 years and above is projected to increase from 1.9 million in 2012 to 17.8 million in 2050 . The elderly population forms a big slice of the overall pharmaceutical spending in the GCC and will also drive growth [10].

Vision 2030 that was recently launched in Saudi Arabia is a great motive to advance Pharmaceutical Industry as it will enforce health insurance, privatize/corporatize the healthcare in Saudi Arabia and invites foreign investors in all aspects of the industry including Pharmaceuticals. This came with great governmental support for the foreign investors. Lands in industrial area are given free to the investors, as well as tax break for a number of years. This will enhance the growth of the industry as well as it will promote creating more jobs for the Nationals and foreign workers.

The great concern is that most the Pharmaceuticals manufacturers in the GCC countries are producing generics, yet the market is open for the international investors, I believe that most, if not all, these international companies will be competing with Generics. I believe that they are here to manufacture their own patented products and may be some generics. It is not clear if the original Saudi Pharmaceutical companies will be able to stand the competition, or stay in the market. Their philosophy had to change to stay.

The demand for pharmaceuticals is currently witnessing robust growth in Saudi Arabia, mainly due to increasing prevalence of noncommunicable diseases and improved healthcare infrastructure. The pharmaceuticals market in Saudi Arabia is mainly dominated by multinational companies, which account for the largest share in the market. Rising health awareness and increased healthcare 
expenditure are factors expected to drive market growth in the near future. Macroeconomic factors such as increasing penetration of health insurance companies and healthcare reforms such as allowing $100 \%$ Foreign Direct Investment (FDI) in the pharmaceuticals sector is further projected to drive the growth of the market [11].

Saudi Arabia, being the largest market in GCC region, presents better investment opportunities for investors, and this is further augmented with mandatory insurance and corporatization / privatization of the healthcare sector in the country. However, lack of indigenous research capabilities, delays in patent registration, and lack of trained Saudi workers are major factors expected to hamper overall growth of the Saudi Arabia pharmaceutical market over the forecast period.

\section{Conclusion}

Pharmaceutical Industry is an essential industry in any country. GCC countries have taken the initiative to start this valuable industry more than 30 years ago. Yet the outcome after these years, they are still importing $90 \%$ of their needs from the international manufacturers. I am confident that the GCC governments when they funded and supported this industry, they were hoping to be self-sufficient within 30 years, not manufacturing only $10 \%$ of their needs and they are all competing for the same products.

In my view, the new era of Pharmaceutical industry development should be regulated in a manner which assures quality, integration and development of an R\&D within the new Pharmaceutical companies. Also another factor which will assure the success of the industry is the training of the nationals to understand and practice the industry. Pharmacy schools should cater their curriculum to provide the necessary education and training to build the new workforce for this industry. This industry doesn't need a lot of Pharmacist but a lot of technical personnel. Training institutes for allied health also should take a lead in providing the necessary training as deemed necessary by the industry. Most of the highly educated Pharmacists provide education in the university, and they are providing limited laboratory research. The Universities has a social responsibility to support R\&D and require that their staff should be involved in research and development as part of their career. We have a huge man force that can stand up the challenge of the new era, however, they are underutilized their main task is teaching the basic academic courses. The very same individuals were involved in research for major Pharmaceutical companies during their study abroad.

For the business men, who are investing in the Pharmaceutical industry, they should have a broader vision for the future, they should encourage and support $\mathrm{R} \& \mathrm{D}$ because the outcome of developing a new molecule or formulation will be greatly rewarding. It takes patience and a lot of investment as well as comprehensive research to reach the goal.

Biotechnology, should be given priority in the new era development, as Biotechnology is the future of medicine, GCC countries should give it priority and support. It is part of the national security for the country as well as it is the new treatment modalities for all diseases. If it was ignored at this stage we will be facing the same situation with relaying on the multinational companies in the future.

With new regulations and the Vision that the GCC countries have set, I feel optimistic for brighter future of the Pharmaceutical Industry in the GCC countries.

\section{References}

1. https://www.zawya.com/mena/en/story/GCC_to_spend_USD12bn_on_ pharmaceutical_until_2020-ZAWYA20131128080750/.

2. Alpen Capital Investment Banking (2013) GCC Pharmaceutical Sector.

3. Capital AM (2014) MENA Healthcare Sector.

4. B.M. International "Country-wise market size Pharmaceutical \& healthcare reports," 2012 .

5. https://www.clydeco.com/insight/article/beyond-oil-the-pharmaceutical-industry-inthe-uae?utm_source $=$ Mondaq\&utm_medium $=$ syndication\&utm_campaign $=$ ViewOriginal.

6. http://www.ebpf-uae.com/.

7. http://www.julphar.net/gcc-could-be-biotech-hub.

8. Dolitte, "Global life sciences outlook, Thriving in today's uncertain market," 2017.

9. http://www.english.globalarabnetwork.com/GCC-Business/gcc-countries-import-90of-pharmaceutical-needs.html.

10. Saudi Gazette, Tuesday July 18, 2017 / 24 , Shawwal , 1438, July 18th 2017.

11. F. M. I. (FMI), "Saudi Arabia Pharmaceutical Market: Supportive Government Reforms and Rising Diseases Awareness to Fuel Revenue Growth from Branded Pharmaceuticals in Saudi Arabia: Industry Analysis and Opportunity Assessment, 2016-2026," 2016.

Copyright: (C2017 Alsaddique A. This is an open-access article distributed under the terms of the Creative Commons Attribution License, which permits unrestricted use, distribution, and reproduction in any medium, provided the original author and source are credited. 